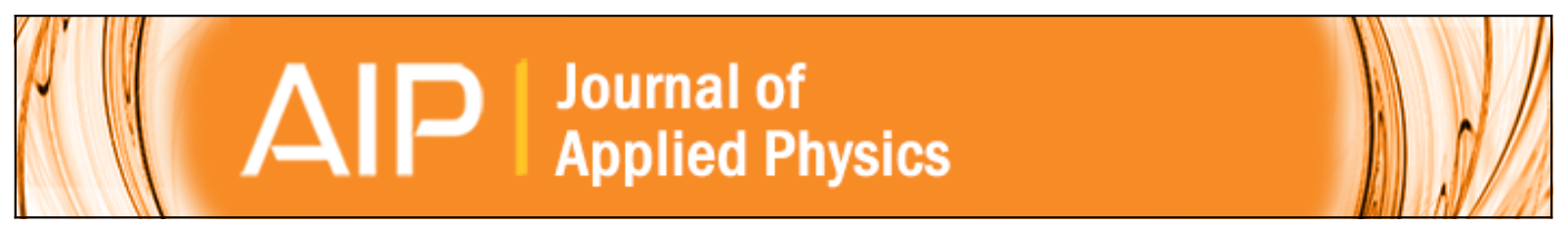

Shock pressure induced by glass-confined laser shock peening: Experiments, modeling and simulation

Xianqian Wu, Zhuping Duan, Hongwei Song, Yanpeng Wei, Xi Wang, and Chenguang Huang

Citation: Journal of Applied Physics 110, 053112 (2011); doi: 10.1063/1.3633266

View online: http://dx.doi.org/10.1063/1.3633266

View Table of Contents: http://scitation.aip.org/content/aip/journal/jap/110/5?ver=pdfcov

Published by the AIP Publishing

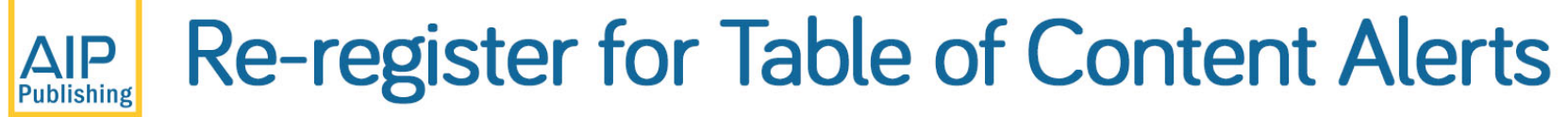




\title{
Shock pressure induced by glass-confined laser shock peening: Experiments, modeling and simulation
}

\author{
Xianqian Wu, ${ }^{1}$ Zhuping Duan, ${ }^{2}$ Hongwei Song, ${ }^{1, a)}$ Yanpeng Wei, ${ }^{1}$ Xi Wang, ${ }^{1}$ \\ and Chenguang Huang ${ }^{1, b)}$ \\ ${ }^{1}$ Key Laboratory for Hydrodynamics and Ocean Engineering, Institute of Mechanics, Chinese Academy of \\ Sciences, Beijing 100190, People's Republic of China \\ ${ }^{2}$ The State Key Laboratory of Nonlinear Mechanics, Institute of Mechanics, Chinese Academy of Sciences, \\ Beijing 100190, People's Republic of China
}

(Received 16 March 2011; accepted 3 August 2011; published online 15 September 2011)

\begin{abstract}
The shock pressure generated by the glass confined regime in laser shock peening and its attenuation in the target material are investigated. First, the particle velocity of the target back free surface induced by laser generated shock pressure of this regime is measured using a photonic Doppler velocimetry system. The temporal profile of the particle velocity at the back free surface, where the elastic precursor is captured, manifests a powerful diagnostic capability of this newly developed photonic Doppler velocimetry system for tracking the velocity on short time scales in shock-wave experiments. Second, a coupling pressure analytical model, in which the material constitutive models of confined layers and target material are considered, is proposed to predict the plasma pressure profile at the surface of target. Furthermore, using the predicted shock pressure profile as the input condition, the dynamic response of the target under the shock pressure is simulated by LS-DYNA. The simulated back free surface velocity profile agrees well with that measured by the photonic Doppler velocimetry system. Finally, the attenuation behavior of stress waves and particle velocities in the depth of the target is analyzed, and it indicates an exponential decay. The corresponding empirical formulas for the attenuation behavior are given based on the numerical results. C 2011 American Institute of Physics. [doi:10.1063/1.3633266]
\end{abstract}

\section{INTRODUCTION}

Laser shock peening (LSP) is an advanced surface treatment technique, which has been successfully applied to improve the fatigue, corrosion, and wearing resistance of metallic components. ${ }^{1-6}$ Through the interaction of a pulsed high-intensity laser beam and an energy absorption layer on the metallic target surface, a shock wave with a high amplitude can be generated and propagates into the target. ${ }^{2,7-13}$ If the amplitude of the shock wave exceeds the Hugoniot elastic limit (HEL) of the target material, plastic deformation occurs and residual compressive stresses are induced near the surface, which results in the enhancement of fatigue life.

The evaluation of the mechanical effects of LSP needs a comprehensive understanding of the shock pressure and its propagation behavior. There are many studies focused on the characterization of a laser induced shock pressure profile with theoretical analyses and experimental measurements. Some analytical models of shock pressure for LSP were developed. $7,8,14-22$ However, in the previous models, the dynamic effect of the material system was simplified as constant reduced shock impedance. As a result, the dynamic response of the material was not fully considered and a more realistic pressure model is demanded. In the experimental measurement, there are several techniques used for estimating the shock pressure, such as a quartz crystal transducer, ${ }^{23,24}$ polyvinylidene fluoride (PVDF), ${ }^{25}$ and velocimetry

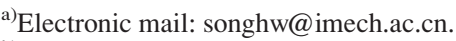

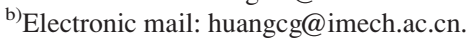

interferometer system for any reflector (VISAR). ${ }^{26} \mathrm{~A}$ quartz crystal transducer is not suitable in LSP because of high amplitude and nanosecond duration of the shock pressure in LSP. ${ }^{8}$ The PVDF is unlikely to work on nanosecond shock measurement because of the limitation of the gauge thickness and impedance mismatch effect. Moreover, it is difficult to calibrate, and the noise signal is difficult to eliminate. The VISAR provides non-contact diagnostics of high resolution, but it has a limitation in tracking low velocities on short time scales, and it is difficult to handle multiple simultaneous velocity measurements. $^{27}$

In the present research, the shock pressure induced by glass-confined LSP is measured with a newly developed photonic Doppler velocimetry (PDV). ${ }^{27-29}$ Compared with water-confined LSP, glass-confinement generates much higher pressure which can be easily reproduced. Since its invention, the use of PDV has been rapidly spreading as a powerful diagnostic method for tracking velocity on the short time scales in shock-wave experiments ${ }^{29}$ and other related areas. ${ }^{30}$ It excels over the VISAR in many aspects, such as low cost, ease of fabrication, and convenience of handling. Actually, PDV can be an ideal replacement in situations where VISAR diagnostics function poorly, such as low velocity and multiple simultaneous velocity measurements. ${ }^{27}$ The problem for PDV, perhaps, is the temporal resolution, and it is reported that PDV has no better temporal resolution than VISAR. ${ }^{31}$ Enough signal beats must be captured for the velocity to be determined. Therefore, there is a limitation on how well the technique works when the velocity of low magnitude is rapidly changing. However, in the 
present study, the elastic precursor wave of LSP is tracked. Moreover, to investigate the shock pressure and its propagation behavior, a more realistic coupling analytical model in conjunction with the finite element method for shock pressure analysis was performed, in which the effect of the material's dynamic response and the thickness of each layer are considered. The attenuations of shock pressure and particle velocity are studied and validated with experimental results.

The article is organized as follows. In Sec. II, the experimental setup and the back free surface velocity measurement technique are described. In Sec. III, the investigation of shock pressure and its propagation behavior is presented. A coupling analytical model for shock pressure profile was developed and the attenuations of shock pressure and particle velocity are studied.

\section{EXPERIMENTS}

\section{A. Laser}

The LSP experiment was performed with a Q-switched high power Nd: YAG pulse laser operating at $1064 \mathrm{~nm}$ wavelength and a maximum of $4 \mathrm{~J}$ output energy per shot can be achieved through a two-step amplification system. A typical temporal profile of the laser pulse, which is in the near Gaussian shape, is recorded as in Fig. 1. The full width at half maximum (FWHM) is approximately $7.32 \mathrm{~ns}$, and the output energy is about $2.68 \mathrm{~J}$. The laser beam is focused with a $600 \mathrm{~mm}$ focal lens to obtain the desired shot radius, and its spatial profile is modulated to a nearly flat shape.

\section{B. Target}

The target material is 1-mm-thick 2024Al. The target surface is glued with a $0.1-\mathrm{mm}$-thick $\mathrm{Al}$ foil as an absorption layer, confined by a 4-mm-thick K9 glass against the laser irradiation. The K9 glass is fully-clamped with the target without a cushion at the back surface by a specially designed hold.

\section{Measurements}

The target back free surface velocity measurement was implemented with a PDV system illustrated in Fig. 2. When the laser illuminates the moving surface to be measured through a probe, a fraction of light is reflected at the interface between the probe lens and air, and acts as the reference light with the original laser frequency of $f_{0}$. The same probe collects the light reflected from the moving surface and sends the signal light with the Doppler-shifted frequency of $f_{b}$ to the detector. The difference in the two frequencies, which is the beat frequency, $\bar{f}_{b}(t)$, can be recorded with a detecting system with enough high bandwidth. The velocity of the moving surface can be obtained by

$$
u(t)=\frac{\lambda_{0}}{2} \bar{f}_{b}(t),
$$

where $\lambda_{0}$ is the original wavelength of the laser.

A CQF938 series high power $1550 \mathrm{~nm}$ continuous wave distributed feedback laser with a polarization maintaining

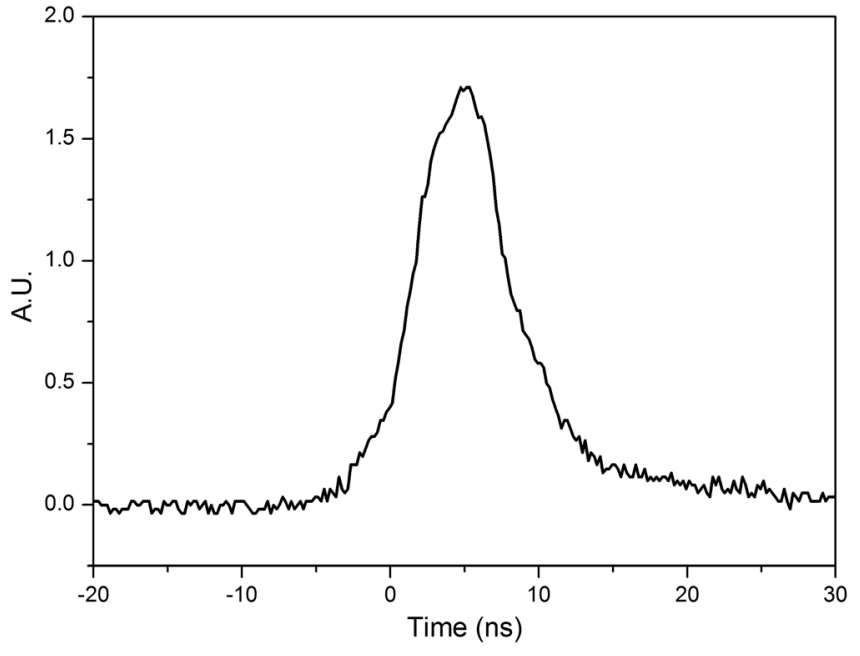

FIG. 1. Laser temporal profile.

fiber, provided by JDS Uniphase Corporation, was used. The laser operated at a power of about $40 \mathrm{~mW}$, and a narrow linewidth of about $200 \mathrm{kHz}$. An InGaAs PIN photodiode detector (provided by New Focus Inc.) is employed, and the bandwidth is $12 \mathrm{GHz}$. The interference signals are recorded with the Lecoy WaveMaster 808Zi oscilloscope operating with the bandwidth of $8 \mathrm{GHz}$ and a sampling rate of $40 \mathrm{Gs} / \mathrm{s}$. To distinguish the interference signal at the very beginning of the target motion, the digital oscilloscope is triggered by the temporal domain of a high power laser pulse with a $\mathrm{Si}$ biased detector. Once triggered, the laser energy per shot, temporal distribution of the laser pulse, and the target back free surface velocity were measured simultaneously and recorded in the oscilloscope.

\section{PDV Results}

A typical measured signal and the calculated back free surface velocity are shown in Fig. 3. According to Eq. (1), the instantaneous velocity is proportional to the beat frequency, $\bar{f}_{b}(t)$. Therefore, the density of interference fringes indicates velocity magnitude; the closer the two adjacent fringes are, or the higher the beat frequency $\bar{f}_{b}(t)$ is, the faster the velocity at the moment is. The oscillation of the velocity is due to the reflection of the shock wave at the surfaces of the target, and the duration of the adjacent peak velocity is about two times that of the transmission duration

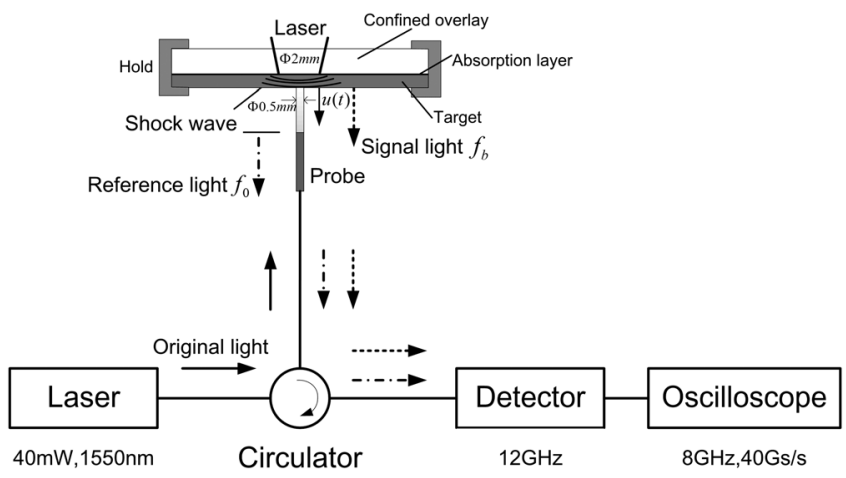

FIG. 2. Configuration of the PDV. 
of the shock wave through the target. The zero point at the horizontal coordinate is the onset of the laser pulse, which has been illustrated in Fig. 1. The first two interference fringes, starting at about $175 \mathrm{~ns}$, are caused by an elastic precursor wave, indicating the propagation of the elastic wave reaches the back free surface at this time. The back free surface velocity caused by the elastic precursor wave is about $57.9 \mathrm{~m} / \mathrm{s}$. At $224.8 \mathrm{~ns}$, the surface velocity reaches the first peak value of about $313.9 \mathrm{~m} / \mathrm{s}$, indicating that the first shock wave reaches the back surface. At $553.8 \mathrm{~ns}$, the surface velocity is about $153.5 \mathrm{~m} / \mathrm{s}$ when the shock wave reaches the back surface again. The time duration of the adjacent peak velocity is about $329 \mathrm{~ns}$.

According to the Rankin-Hugoniot relations, ${ }^{32,33}$ for the uniaxial strain state, the compressive pressure, $p$, can be written as Eq. (2) while $p$ is smaller than HEL,

$$
p=\rho_{0} C_{e} u .
$$

If $p$ exceeds HEL,

$$
p=\rho_{0}\left(C_{0}+S u\right) u+\frac{2}{3} Y_{0},
$$

where $C_{e}=6.41 \times 10^{3} \mathrm{~m} / \mathrm{s}$ is the elastic stress wave velocity, $\rho_{0}=2.77 \times 10^{3} \mathrm{~kg} / \mathrm{m}^{3}$ is the initial material density, $C_{0}=5.33 \times 10^{3} \mathrm{~m} / \mathrm{s}$ is the sound velocity at zero pressure, $S=1.34$ is the empirical material parameter, $u$ is the particle velocity, $Y_{0}=0.265 \mathrm{GPa}$ is the yield stress for the $2024 \mathrm{Al}$ material, respectively, ${ }^{33,34}$ and $\mathrm{HEL}=(1-\nu) Y_{0} /(1-2 \nu)$ $=0.547 \mathrm{GPa}$.

According to Eq. (2), the elastic particle velocity is about $30.80 \mathrm{~m} / \mathrm{s}$, which is approximately half of the experimentally measured maximum velocity caused by the elastic precursor wave at the free surface.

When the target back free surface velocity, $u_{\text {surf }}$, is measured by PDV, the shock pressure at the back surface position for an infinite target can be determined by Eqs. (2) or (3), where $u=u_{\text {surf }} / 2$ to take into account the reflection of stress wave at the free surface.

\section{SHOCK PRESSURE ANALYSIS}

\section{A. Modeling}

Several analytical models have been developed to predict the shock pressure profile in the LSP process. ${ }^{7,14-16,19-22,35,36}$ According to the laser irradiation time, the confined plasma will endure three typical stages including laser heating, adiabatic cooling, and impulse moment translation. ${ }^{7}$ In the last stage, the pressure is too low to cause a plastic deformation of the material. Therefore, the first two stages of the shock pressure profile are considered. In the present research, the pressure analysis is based on a new coupling analysis model, in which the effect of the material's dynamic response and the thickness of the confined overlay, absorption layer, and target material are considered.

The one-dimensional coupling analytical model for shock pressure induced by LSP is shown in Fig. 4. The parameters, $h_{A}, u_{A, e v}, u_{A}$, and $h_{C}, u_{C, e v}, u_{C}$ are thickness, laser ablation particle velocity, stress related particle velocity for

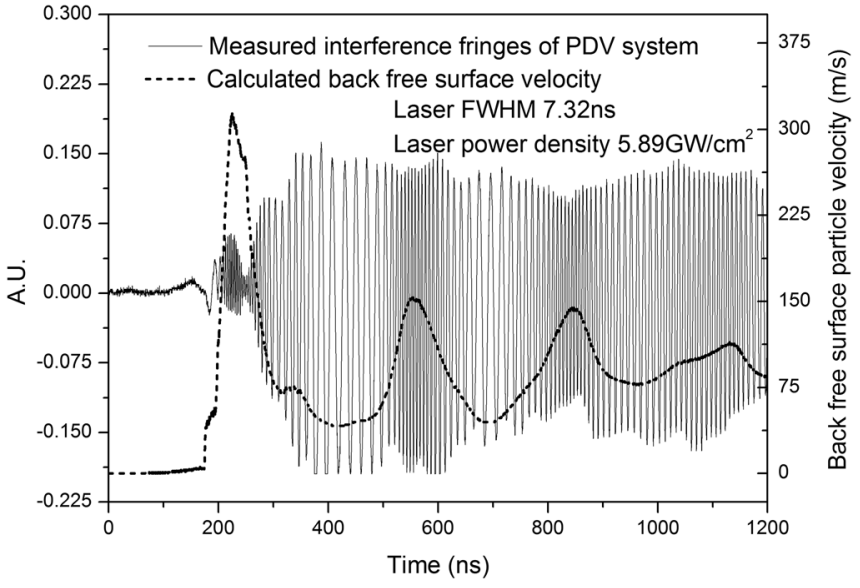

FIG. 3. Interference fringes measured by the PDV system and corresponding back free surface velocity profile.

absorption layer (subscript A) and confined overlay (subscript C), respectively. Here, $\sigma_{C}=g_{C}\left(\varepsilon_{C}, \dot{\varepsilon}_{C}\right)$, $\sigma_{A}=g_{A}\left(\varepsilon_{A}, \dot{\varepsilon}_{A}\right)$, and $\sigma_{T}=g_{T}\left(\varepsilon_{T}, \dot{\varepsilon}_{T}\right)$ are the material constitutive models for the confined overlay, absorption layer, and target material, respectively. The thickness of the target is $h_{T}$. The pressure, $p(t)$, and length, $L(t)$, with an initial value of $L_{0}$ of the plasma vary with its state at different times. The fraction of the internal energy converted to the thermal energy is $\alpha$ and typically is $0.25{ }^{7,8}$ The incident laser power density is $I_{0}(t)$, and $R_{C}$ and $R_{P}$ are the laser reflection coefficients of the confined overlay's surfaces as shown in Fig. 4. Here, $\kappa_{p}$ and $\kappa_{C}$ are the laser absorption coefficients for plasma and confined overlay.

Assuming the surface velocity is composed of laser ablation velocity and stress related velocity, ${ }^{15,35}$ the continuity condition of the plasma can be written as,

$$
\frac{d L(t)}{d t}=u_{C}+u_{C, e v}+u_{A}+u_{A, e v} .
$$

According to Fabbro et al., ${ }^{7}$ one portion of the laser energy absorbed by the plasma contributes to the work of the confined material, and the other converts to its internal energy used for the ionization and thermal energy increasing of the plasma. Taking the plasma as an ideal gas, while laser irradiating, ${ }^{7}$

$$
I_{p}(t)=p(t) \frac{d L(t)}{d t}+\frac{3}{2 \alpha} \frac{d}{d t}[p(t) L(t)]
$$

where $I_{p}(t)$ is the absorbed energy power density,

$$
\begin{aligned}
I_{p}(t)= & I_{0}(t) \cdot\left(1-R_{C}\right) \cdot \exp \left(-\kappa_{C} h_{C}\right) \cdot\left(1-R_{p}\right) \\
& \cdot\left[1-\exp \left(-\kappa_{p} L\right)\right] .
\end{aligned}
$$

After the laser is switched off, the confined plasma is supposed to experience an adiabatic cooling stage. Consequently,

$$
p(t)=p(\tau)\left(\frac{L(\tau)}{L(t)}\right)^{\gamma}
$$

where $\tau$ is the laser pulse duration, $L(\tau)$ and $p(\tau)$ are the thickness and pressure of the plasma when the laser is switched off, and $\gamma$ is the adiabatic exponent of the plasma. 


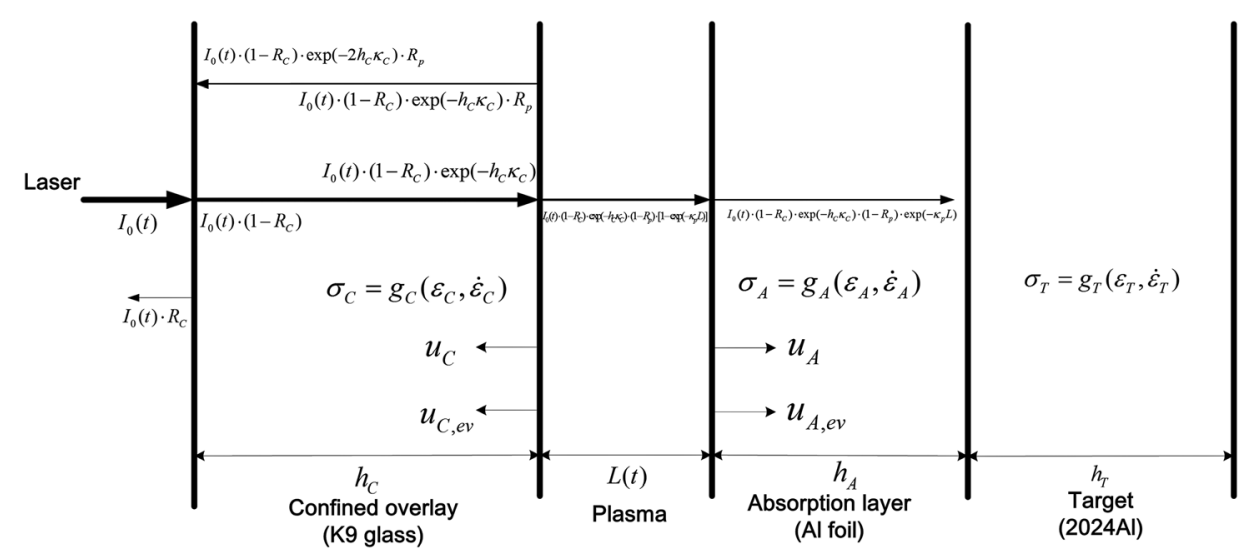

FIG. 4. One-dimensional coupling analytical model for shock pressure.
The laser ablation velocities, $u_{C, e v}$ and $u_{A, e v}$, are calculated as the stable evaporation model, ${ }^{37}$

$$
u_{C, e v}=\frac{I_{0}(t)\left(1-R_{C}\right)\left[1-\exp \left(-\kappa_{C} h_{C}\right)\right]\left[1-R_{p} \exp \left(-\kappa_{C} h_{C}\right)\right]}{\rho_{C}\left[L_{C, c}+L_{C, n}+C_{C}\left(T_{C, c}-T_{C, n}\right)\right]},
$$

$$
u_{A, e v}=\frac{I_{0}(t)\left(1-R_{C}\right) \exp \left(-\kappa_{C} h_{C}\right)\left(1-R_{p}\right) \exp \left(-\kappa_{p} L\right)}{\rho_{A}\left[L_{A, c}+L_{A, n}+C_{A}\left(T_{A, c}-T_{A, n}\right)\right]},
$$

where $\rho_{C}, L_{C, c}, L_{C, n}, C_{C}, T_{C, c}, T_{C, n}$ and $\rho_{A}, L_{A, c}, L_{A, n}$, $C_{A}, T_{A, c}, T_{A, n}$ are the density, latent heat of fusion, latent heat of vaporization, specific heat, melting point, boiling point for the confined overlay and the absorption layer, respectively.

The stress related velocities are dependent on the constitutive model and thickness of the material by considering the reflection of the stress waves at the free surface of the confined overlay and the interface of the absorption material and target. While the materials yield, the interaction of the elastic and plastic stress wave is complex. As a result, the stress related velocities are a little difficult to determine. We supposed that the stress related velocities are functions of the plasma's pressure, $p$, and its change rate, $d p / d t$, where the pressure $p$ and its change rate $d p / d t$ represent the stress and strain rate the materials experienced.

$$
\begin{aligned}
& u_{C}=f_{C}\left(p, d p / d t, h_{C}, m_{C}\right), \\
& u_{A}=f_{A}\left(p, d p / d t, h_{A}, m_{A}\right),
\end{aligned}
$$

where $m_{C}$ and $m_{A}$ represent the constitutive model related to the parameters of the confined overlay and absorption layer, respectively.

Equations (4) to (11) need to be solved simultaneously because they are coupled with each other. When the incident laser power density, $I_{0}(t)$, is given, the shock pressure can be analyzed. In the present research, the stress related velocities are calculated by the finite element package LS-DYNA ${ }^{38}$ for the appropriate numerical model at each time step. Therefore, the explicit difference method and finite element simulation are combined to obtain the pressure profile.

Compared with the previous models, ${ }^{7,8,14-18}$ it can be found that the model in the present research is more physical and takes more factors into account. The expansion velocity of the plasma is associated with not only the applied pressure state and material constitutive models of the confined overlay, absorption layer, and target, but also the geometry sizes, such as the thicknesses of the confined overlay and absorption layer, to take the influence of the free surface boundary into account. However, the ablation velocities of the confined overlay and absorption layer are simplified in the present analysis model, and the variable $\alpha$ is uncertain, which can be determined by considering the laser ablation and the evolution process of the plasma. In addition, more experiments of the glass-confined LSP process will be performed to validate the model in the future.

\section{B. Simulations}

The dynamic response of the 2024Al material was simulated with the explicit dynamic algorithm of the LS-DYNA finite element software package. The LS-DYNA code has been validated by $\mathrm{Hu}$ et $a l .{ }^{39}$ to simulate the LSP process. The applied pressure at each loading step was calculated with the analytical method in Sec. III A, where the elastic constitutive model was taken for K9 glass. As the K9 glass does not have a clear melting point, the critical temperature, $\mathrm{T}_{\mathrm{R}}$, is employed to calculate the laser ablation velocity. The material properties of $\mathrm{K} 9$ glass is given in Table I. The Johnson-Cook constitutive model was used to described material dynamic behavior for $\mathrm{Al}$ foil and 2024Al, and the effective stress can be written as

$$
\sigma_{\gamma}=\left(A+B \bar{\varepsilon}^{n}\right)\left(1+C 1 n \dot{\varepsilon}^{*}\right)
$$

TABLE I. Material properties for K9 glass (Refs. 42, 43).

\begin{tabular}{lc}
\hline \hline Material & K9 glass \\
\hline Density, $\mathrm{kg} \mathrm{m}^{-3}$ & 2520 \\
Young's modulus, GPa & 82.3 \\
Poisson's ratio & 0.209 \\
Laser reflection coefficient & 0.035 \\
Laser absorption coefficient, $\mathrm{cm}^{-1}$ & 0.01 \\
Specific heat, $\mathrm{J} \mathrm{g}^{-1} \mathrm{~K}^{-1}$ & 0.71 \\
Critical temperature, $\mathrm{K}$ & 1100 \\
\hline \hline
\end{tabular}




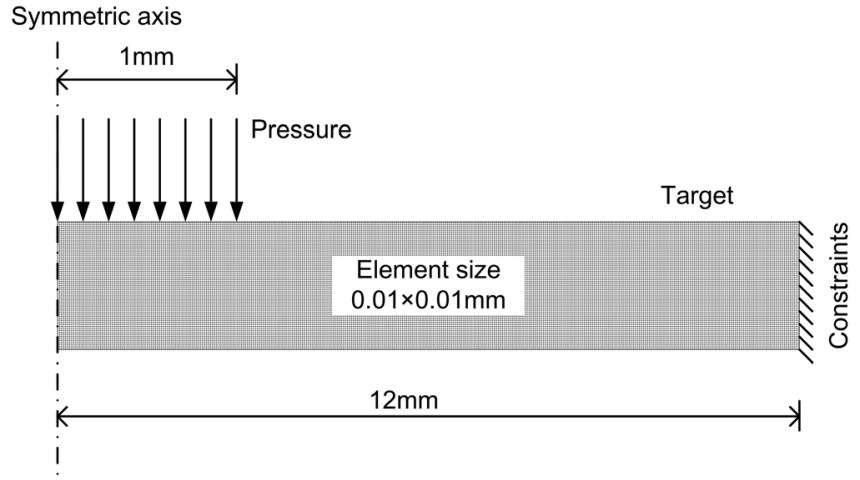

FIG. 5. Numerical model.

where $\overline{\varepsilon^{p}}$ is the equivalent plastic strain; $\dot{\varepsilon} *=\varepsilon^{p} / \dot{\varepsilon}_{0}$ is normalized equivalent plastic strain rate and $\dot{\varepsilon}_{0}=1 \mathrm{~s}^{-1}$, A, B, and $\mathrm{C}$ are material constants, and $\mathrm{n}$ is the work hardening exponent.

A two-dimensional axisymmetric finite element model was built to analyze the back free surface velocity and depicted in Fig. 5. The FE model represents a cylindrical target with a circular shaped laser spot irradiation. The edge (right boundary) of the circular target is fully constrained and the bottom surface is free. The analyzed pressure profile is applied on the laser irradiated region of the upper boundary. The model is finely meshed with $2 \mathrm{D}$ solid elements in the size of $0.01 \times 0.01 \mathrm{~mm}$. The material properties for $\mathrm{Al}$ foil $^{8}$ and 2024Al (Refs. 33, 34) used in the simulation are shown in Table II. The simulated surface velocity and the experimentally measured result are shown in Fig. 6. As the formation and evolution of the plasma are not taken into account in the coupling analytical model, the delay effect of the analyzed pressure profile will be neglected. The simulation results, including the back surface velocity profile and time durations $\tau_{0}$ of the adjacent peak velocity, fit well with the experimental results before the first $1200 \mathrm{~ns}$, which showed the consistency of the numerical model. However, the simulated elastic precursor velocity of the back free surface is a little higher than the experiment value. It might be ascribed to the parameters of the material constitutive model, which is determined by relatively low strain rate experiments. In the LSP process, the strain rate can reach $10^{7} \mathrm{~s}^{-1}$,

TABLE II. Material properties for Al foil (Ref. 8) and 2024Al (Refs.33, 34).

\begin{tabular}{lcc}
\hline \hline \multicolumn{1}{c}{ Material } & Al foil & $2024 \mathrm{Al}$ \\
\hline Density, $\mathrm{kg} \mathrm{m}^{-3}$ & 2700 & 2770 \\
Young's modulus, GPa & 52.03 & 73.84 \\
Poisson's ratio & 0.39 & 0.34 \\
Parameter A, MPa & 200 & 265 \\
Parameter B, MPa & 426 & 426 \\
Parameter C, MPa & 0.015 & 0.015 \\
Parameter n & 0.34 & 0.34 \\
Specific heat, J g ${ }^{-1} \mathrm{~K}^{-1}$ & 0.875 & 0.875 \\
Latent heat of fusion, $\mathrm{J} \mathrm{g}^{-1}$ & 398 & 398 \\
Latent heat of vaporization, $\mathrm{J} \mathrm{g}^{-1}$ & 10900 & 10900 \\
Melting point, $\mathrm{K}$ & 933 & 933 \\
Boiling point, $\mathrm{K}$ & 2792 & 2792 \\
\hline \hline
\end{tabular}

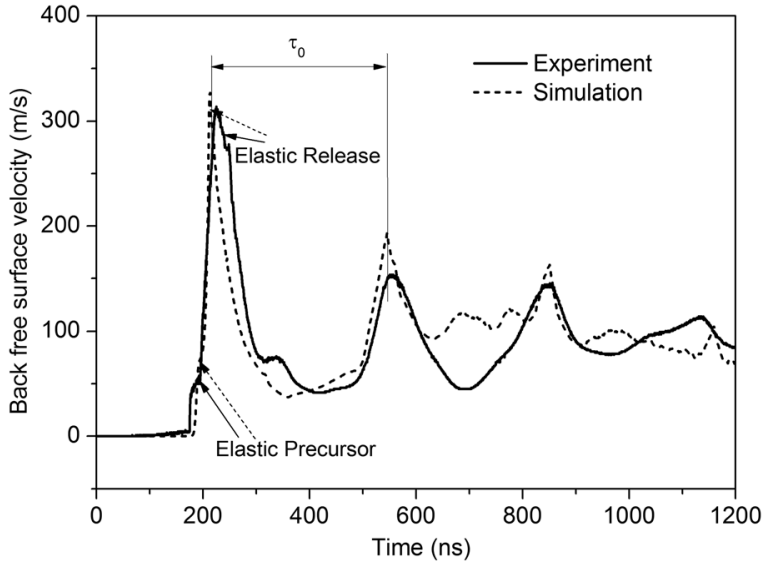

FIG. 6. The back free surface velocities that are simulated and measured by the PDV system.

and the mechanical properties of the material change significantly at that condition compared with the relatively low range of strain rate. ${ }^{33}$

To analyze the attenuation of the stress waves and particle velocity, a semi-infinite simulation model was developed. The decay of the shock waves in depth is presented in Fig. 7 for different incident laser power density with a FWHM of $7.32 \mathrm{~ns}$. The applied pressure profile is given by the same method. Since the elastic stress wave is faster than the plastic stress wave, the elastic precursor obviously breaks off ahead of the plastic wave. In addition, the decay of shock waves for the relatively higher incident laser power density is faster than the lower one, due to the higher strain rate and the faster increase of the plastic strain energy. The stress waves and particle velocities at different depths of the impact center are shown in Figs. 8 and 9, where the increment of depth for adjacent curves $\delta H$ is $0.3 \mathrm{~mm}$. The attenuation behaviors of the stress waves and particle velocities in depth manifest similar tendency, and the peak values indicate an approximately exponential decay. The attenuation behaviors of the stress waves and particle velocities are much more complex and related to the material constitutive, peak value of the shock wave, $p_{m}$, or the particle velocity at the impact center, $u_{m}$, laser diameter, $2 R$, and the depth from the impact surface, $H$.

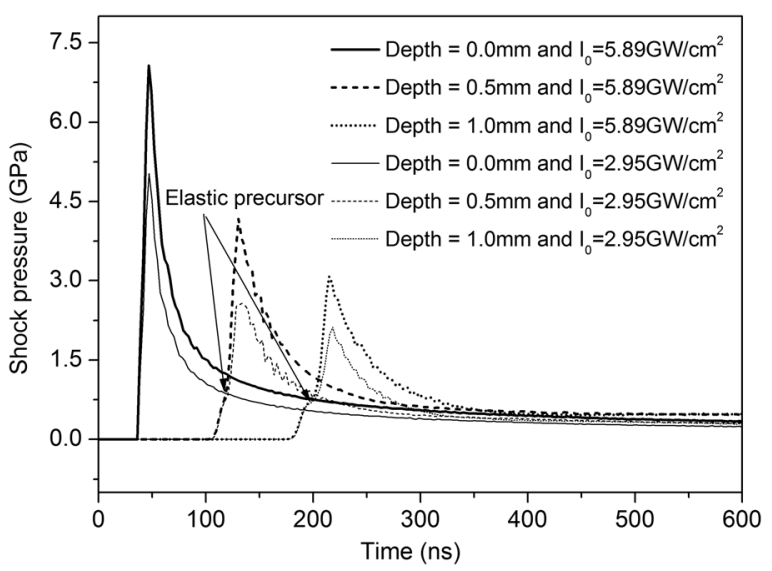

FIG. 7. The decay of the shock waves in depth for different peak laser power densities. 


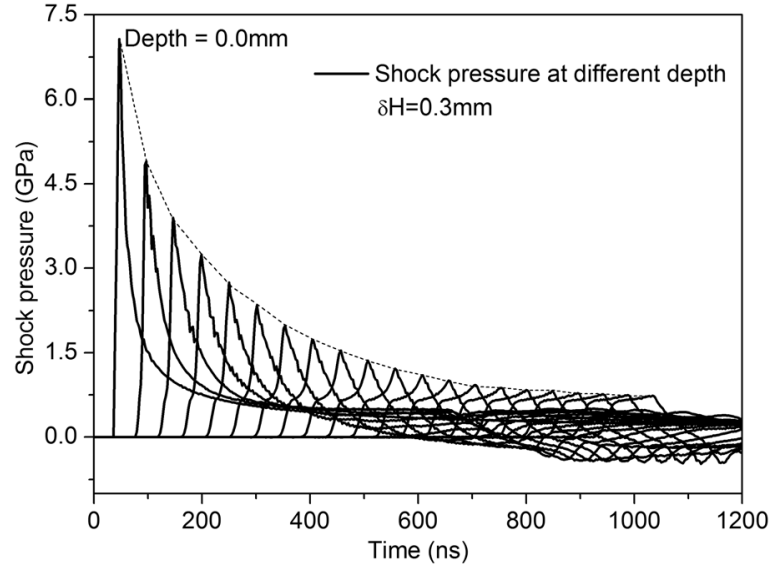

FIG. 8. The stress waves at different depths of the impact center.

While the material is unchanged, the shock wave amplitude, $p$, and particle velocity, $u$, at depth, $H$, are related using dimensional analysis ${ }^{40,41}$ as follows:

$$
\begin{aligned}
& p / p_{m}=f(H /(2 R)), \\
& u / u_{m}=f^{\prime}(H /(2 R)) .
\end{aligned}
$$

The attenuation of the normalized shock wave amplitude, $p / p_{m}$, and the normalized particle velocity, $u / u_{m}$, in normalized depth, $H /(2 R)$, are illustrated in Fig. 10. To describe the propagation of the stress wave in the target, the attenuation of $p / p_{m}$ and $u / u_{m}$ with $H /(2 R)$ could be fitted based on the numerical results as follows:

$$
\begin{aligned}
p / p_{m}= & 0.67 \exp \left[-\frac{H /(2 R)}{0.71}\right]+0.25 \exp \left[-\frac{H /(2 R)}{0.12}\right] \\
& +0.09 \\
u / u_{m}= & 0.70 \exp \left[-\frac{H /(2 R)}{0.72}\right]+0.23 \exp \left[-\frac{H /(2 R)}{0.12}\right] \\
& +0.08 .
\end{aligned}
$$

Therefore, the attenuation of shock pressure and particle velocity can be described throughout the depth if the applied pressure profile is given. It can also be realized that the attenuations of the stress wave and particle velocity are not

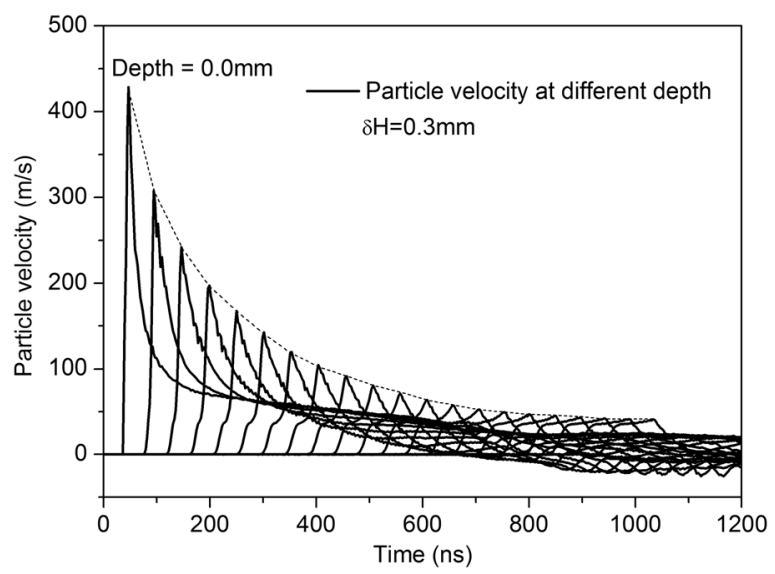

FIG. 9. The particle velocities at different depths of the impact center.

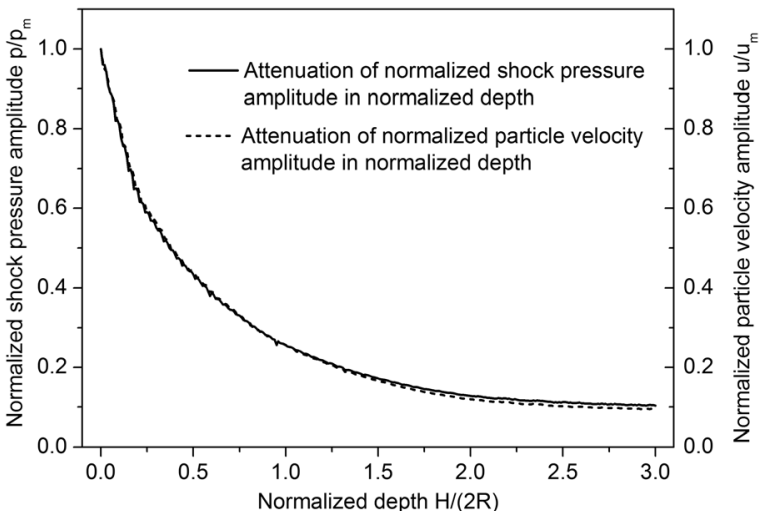

FIG. 10. The attenuation of normalized shock wave amplitude, $\mathrm{p} / \mathrm{p}_{\mathrm{m}}$, and normalized particle velocity, $\mathrm{u} / \mathrm{u}_{\mathrm{m}}$, in normalized depth $\mathrm{H} /(2 \mathrm{R})$.

strictly consistent throughout the normalized depth. This is due to the changes of the mechanical properties in the rate related material while the stress propagates. ${ }^{32}$ From this point of view, a potential method can be provided by the Lagrange method ${ }^{32}$ and PDV measurement to obtain the stress-strain relation of a material under LSP.

\section{SUMMARY}

In this paper, the shock pressure generated by the glass confined regime in the LSP process and its propagation in the target material are investigated by experiments and simulation.

The PDV measurement is performed to track the back free surface velocity of the target on the short time scales in the LSP experiment. The temporal particle velocity profile, including the elastic precursor wave velocity of the back free surface, is tracked, indicating an effective diagnostic method to study the characteristics of shock pressure induced in LSP.

A more detailed coupling pressure analytical model, in which the dynamic responses of the confined overlay, absorption layer, and target material are considered, is proposed to predict the shock pressure profile. This pressure model may be much more approximate to the physical essence.

The attenuations of the shock pressure and related particle velocity are investigated using numerical simulation, and the corresponding dimensionless empirical formulas are given to describe the decay behaviors of the shock pressure and particle velocity in depth.

In the present pressure analytical model, the glue layer and the evolution of the plasma are not taken into account, and the laser ablation process is simplified. A more rigorous analytical model will be proposed, and systematic experiments will be performed to validate the model in this later work. In addition, the stress-strain relation of a material under a high strain rate will be examined by the Lagrange method and PDV measurement in LSP experiments in the future.

\section{ACKNOWLEDGMENTS}

The authors would like to thank the National Natural Science Foundation of China (Grant Nos. 10972228, 
11002150, and 91016025), the Major Program of the National Natural Science Foundation of China (Grant No. 10832011) and the Basic Research Equipment Project of the Chinese Academy of Sciences (Grant No. YZ200930) for financial support. The authors would also like to thank Dr. J. Wang and Prof. C. Q. Wu at Beijing Jiaotong University for their efforts in adjusting and testing of the PDV system.

${ }^{1}$ C. Montross, T. Wei, L. Ye, G. Clark, and Y. Mai, Int. J. Fatigue 24(10), 1021 (2002).

${ }^{2}$ K. Ding and L. Ye, Laser Shock Peening: Performance and Process Simulation, 1st ed. (Woodhead, New York, 2006).

${ }^{3}$ P. Peyre and R. Fabbro, Opt. Quantum Electron. 27(12), 1213 (1995).

${ }^{4}$ J. Z. Lu, L. Zhang, A. X. Feng, Y. F. Jiang, and G. G. Cheng, Mater. Des. 30(9), 3673 (2009).

${ }^{5}$ M. S. Schneider, B. Kad, D. H. Kalantar, B. A. Remington, E. Kenik, H. Jarmakani, and M. A. Meyers, Int. J. Impact Eng. 32(1-4), 473 (2005).

${ }^{6}$ J. Z. Lu, K. Y. Luo, Y. K. Zhang, C. Y. Cui, G. F. Sun, J. Z. Zhou, L. Zhang, J. You, K. M. Chen, and J. W. Zhong, Acta Mater. 58(11), 3984 (2010).

${ }^{7}$ R. Fabbro, J. Fournier, P. Ballard, D. Devaux, and J. Virmont, J. Appl. Phys. 68(2), 775 (1990).

${ }^{8}$ L. Berthe, R. Fabbro, P. Peyre, L. Tollier, and E. Bartnicki, J. Appl. Phys. 82, 2826 (1997).

${ }^{9}$ R. J. Trainor, J. W. Shaner, J. M. Auerbach, and D. W. Phillion, Bull. Am. Phys. Soc. 23(1), 105 (1978).

${ }^{10}$ R. J. Trainor, J. W. Shaner, J. M. Auerbach, and N. C. Holmes, Phys. Rev. Lett. 42(17), 1154 (1979).

${ }^{11}$ R. J. Trainor, N. C. Holmes, R. A. Anderson, E. M. Campbell, W. C. Mead, R. J. Olness, R. E. Turner, and F. Ze, Appl. Phys. Lett. 43(6), 542 (1983).

${ }^{12}$ N. C. Anderholm, Appl. Phys. Lett. 16(3), 113 (1970).

${ }^{13}$ B. P. Fairand and A. H. Clauer, J. Appl. Phys. 50(3), 1497 (1979).

${ }^{14}$ A. Sollier, L. Berthe, P. Peyre, E. Bartnicki, and R. Fabbro, Proc. SPIE 4831, 463 (2003).

${ }^{15}$ B. Wu and Y. C. Shin, J. Appl. Phys. 97(11), 113517 (2005).

${ }^{16}$ W. W. Zhang, Y. L. Yao and I. C. Noyan, J. Manuf. Sci. Eng. 126(1), 10 (2004).

${ }^{17}$ B. X. Wu and Y. C. Shin, J. Manuf. Sci. Eng. 129(1), 117 (2007).

${ }^{18}$ B. X. Wu and Y. C. Shin, J. Appl. Phys. 99(8), 084310 (2006).

${ }^{19}$ J. D. Colvin, E. R. Ault, W. E. King, and I. H. Zimmerman, Phys. Plasma 10, 2940 (2003)

${ }^{20}$ J. D. Colvin, B. W. Reed, A. F. Jankowski, M. Kumar, D. L. Paisley, D. C. Swift, T. E. Tierney, and A. M. Frank, J. Appl. Phys. 101, 084906 (2007).
${ }^{21}$ D. C. Swift, J. T. Gammel, and S. M. Clegg, Phys. Rev. E 69(5), 056401 (2004).

${ }^{22}$ D. C. Swift, T. E. Tierney, R. A. Kopp, and J. T. Gammel, Phys. Rev. E 69(3), 036406 (2004).

${ }^{23}$ D. Devaux, R. Fabbro, L. Tollier, and E. Bartnicki, J. Appl. Phys. 74, 2268 (1993).

${ }^{24}$ N. Anderholm, Appl. Phys. Lett. 16, 113 (1970).

${ }^{25}$ S. Couturier, T. de Ressguier, M. Hallouin, J. Romain, and F. Bauer, J. Appl. Phys. 79, 9338 (1996).

${ }^{26}$ L. M. Barker and R. E. Hollenbach, J. Appl. Phys. 43(11), 466-4675 (1972).

${ }^{27}$ D. H. Dolan and S. C. Jones, Rev. Sci. Instrum. 78(7), 076102 (2007).

${ }^{28}$ O. T. Strand, D. R. Goosman, C. Martinez, T. L. Whitworth, and W. W. Kuhlow, Rev. Sci. Instrum. 77(8), 083108 (2006).

${ }^{29}$ D. H. Dolan, Rev. Sci. Instrum. 81(5), 053905 (2010).

${ }^{30}$ A. R. Valenzuela, G. Rodriguez, S. A. Clarke, and K. A. Thomas, Rev. Sci. Instrum. 78(1), 013101 (2007).

${ }^{31}$ M. I. Kaufman, R. M. Malone, B. C. Frogget, D. L. Esquibel, V. T. Romero, G. A. Lare, B. Briggs, A. J. Iverson, D. K. Frayer, and D. DeVore, presented at the Optical System Alignment and Tolerancing Conference, San Diego, CA, USA, 11 September 2007.

${ }^{32} \mathrm{~A}$. Hopkins and P. Chou, Dynamic Response of Materials to Intense Impulsive Loading, 1st ed. (Air Force Materials Laboratory, New York, 1972).

${ }^{33}$ M. Meyers, Dynamic Behavior of Materials, 1st ed. (Wiley-Interscience, New York, 1994).

${ }^{34} \mathrm{G}$. R. Johnson and W. H. Cook, presented at the Proceedings of the 7th International Symposium on Ballistics, (The Hague, The Netherlands, 19-21 April 1983).

${ }^{35}$ B. X. Wu and Y. C. Shin, J. Appl. Phys. 101(2), 023510 (2007).

${ }^{36}$ R. J. Trainor and Y. T. Lee, Phys. Fluids 25(10), 1898 (1982).

${ }^{37}$ C. R. Phipps, R. F. Harrison, T. Shimada, G. W. York, T. P. Turner, X. F. Corlis, H. S. Steele, L. C. Haynes, and T. R. King, Laser Part. Beams 8(1-2), 281 (1990).

${ }^{38}$ J. O. Hallquist, LS-DYNA3D Theoretical Manual (Livermore Software Technology Corporation, Livermore, 1991).

${ }^{39}$ Y. X. Hu, Z. Q. Yao, and J. Hu, Surf. Coat. Technol. 201(3-4), 1426 (2006).

${ }^{40}$ X. Wu, C. Huang, X. Wang, and H. Song, Int. J. Impact Eng. 38(5), 322 (2011).

${ }^{41}$ Q. M. Tan, Dimensional Analysis, 1st ed. (University of Science and Technology Press, Hefei, 2005).

${ }^{42}$ G. Kanel, S. Razorenov, A. Utkin, H. He, F. Jing, and X. Jin, High Press. Res. 16(1), 27 (1998).

${ }^{43}$ J. Batteh, J. Appl. Phys. 53(11), 8 (1982). 